\title{
La enfermedad de Huntington: una difícil relación entre los enfermos y el derecho a la salud en Colombia
}

\section{Huntington's Disease: A Difficult Relationship between Patients and the Right to Health in Colombia}

\section{A doença de Huntington: uma difícil relação entre os doentes e o direito à saúde na Colômbia}

Luz Estella Varela Londoño, $\mathrm{PhD}^{1 *}$

Clara Victoria Giraldo Mora, $\mathrm{PhD}^{1}$

Jaime León Gañán, PhD²

María Mercedes Arias Valencia, PhD ${ }^{1}$

Recibido: 26 de mayo de $2020 \cdot$ Aceptado: 14 de diciembre de 2020

Doi: https://doi.org/10.12804/revistas.urosario.edu.co/revsalud/a.10288

Para citar este artículo: Varela Londoño LS, Giraldo Mora CV, León Gañán J, Arias Valencia MM. La enfermedad de Huntington: una difícil relación entre los enfermos y el derecho a la salud en Colombia. Rev Cienc Salud. 2021;19(2):1-19.

https://doi.org/10.12804/revistas.urosario.edu.co/revsalud/a.10288

\section{Resumen}

Introducción: el derecho a la salud está consagrado en la Constitución Política y en la Ley Estatutaria de Salud 1751 de 2015. Las personas con enfermedad de Huntington requieren atención especializada e interdisciplinaria, por ser un complejo trastorno genético neurodegenerativo, que comienza en mitad de la vida adulta y no es curable. Materiales y métodos: estudio cualitativo, en diferentes regiones, mediante entrevistas en visita familiar, observación participante y revisión documental. Los resultados son fruto del análisis, realizado según postulados de la teoría fundamentada con origen en la sociología. Resultados: se encontró una difícil relación de las personas con el sistema de salud: los que buscan atención, encuentran múltiples barreras que tratan de superar a través de la tutela, viven en ciudades

1 Facultad de Enfermería, Universidad de Antioquia (Colombia).

2 Facultad de Derecho, Corporación Universitaria Americana (Colombia).

Luz Estella Varela Londoño: orciD https://orcid.org/0000-0002-0652-9402

Clara Victoria Giraldo Mora: oRCiD https://orcid.org/0000-0001-8220-6077

Jaime León Gañan Echavarria: oRciD https://orcid.org/0000-0002-6873-8744.

María Mercedes Arias Valencia: oRciD https://orcid.org/0000-0003-3876-5976

*Autora de correspondencia: luzvarlon@gmail.com, luz.varela@udea.edu.co 
principales o intermedias con alguna atención que los favorezca. Otros, en regiones distantes como Chocó y Juan de Acosta (Atlántico) emprenden pocas acciones en una suerte de "desesperanza aprendida”, como propone Seligman: "no hacer nada porque nada va a funcionar". Conclusión: ellos presentan la enfermedad en estado genuino, no reciben atención y sus condiciones son precarias hasta estados de desnutrición y abandono. La mayoría no tuvo acceso al trabajo formal; por lo tanto, no tendrán posibilidad de la pensión de invalidez o vejez, otros se debaten en el proceso de lograrla, y se encuentran en condiciones de pobreza y precariedad.

Palabras clave: seguridad; social; pensiones; derecho a la salud; enfermedad de Huntington; enfermedad huérfana.

\section{Abstract}

Introduction: The right to health is enshrined in the Political Constitution of Colombia as well as in the Statutory Health Legislation 1751 of 2015. Patients with Huntington's disease require specialized and interdisciplinary care because of the complex genetic neurodegenerative nature of the disorder, which usually affects the middle-aged individuals and is incurable. Materials and methods: This qualitative study was conducted in different regions, followed by data collection through interviews during family visits, participant observation, and documentary review. The results are a part of the analysis, which was conducted according to the grounded theory postulates with an origin in sociology. Results: A difficult relationship was noticed between the individuals and health system, indicating that those seeking care encounter multiple barriers and then attempt to overcome them through tutelage and live in principal or intermediate cities with favorable care availabilities. Others living in remote regions, such as Chocó and Juan de Acosta on the Atlantic, undertake only a few actions as a sort of "learned helplessness," better summarized by Seligman as "doing nothing because nothing will work." Conclusion: The results of the present study indicate that Huntington's disease in the genuine state receives no care and that the conditions of these patients are precarious to the states of malnutrition and abandonment. The majority of these patients have no access to formal employment; thus, they see no possibility for disability pension or retirement, while others debate about the process of achieving it and suffering from poverty and precariousness.

Keywords: Social security; pensions; right to health; Huntington disease; rare diseases.

\section{Resumo}

Introdução: o direito à saúde está consagrado na Constituição Política e na Lei Sanitária Estatutária 1751 de 2015. Pessoas com doença de Huntington requerem atendimento especializado e interdisciplinar por se tratar de uma doença genética neurodegenerativa complexa, que se inicia na metade da vida adulta e não é curável. Materiais e métodos: estudo qualitativo, realizado em diferentes regiões. A coleta foi realizada por meio de entrevistas em visitas familiares, observação participante e revisão documental. Os resultados são fruto de análises, realizadas de acordo com os postulados da teoria fundamentada com origem na sociologia. Resultados: constatou-se a difícil relação entre as pessoas e o sistema de saúde: quem busca atendimento encontra múltiplas barreiras que tentam superar por meio de processo judicial, moram em cidades principais ou intermediárias com algum atendimento que os favorece. Outros, vivem em regiões distantes como Chocó e Juan de Acosta no Atlântico, empreendem poucas tentativas de processo judicial como uma espécie de "desesperança aprendida" como propõe Seligman: "não faça nada porque nada vai funcionar”. Conclusão: os pacientes apresentam a doença de forma genuína, não recebem atenção médica e vivem em condições precárias com certo grau de desnutrição e em estado de abandono. A maioria não teve acesso ao trabalho formal, portanto, não terá a possibilidade de receber aposentadoria por invalidez ou idade, outros estão lutando para obtê-lo, e se encontram em condições de pobreza e precariedade.

Palavras-chave: segurança; social; pensões; direito à saúde; doença de Huntington; doença rara. 


\section{Introducción}

F n Colombia, la salud es un derecho consagrado en la Constitución Política de 1991, espeEcialmente en los artículos 44, 48 y 49 (1). Con relación al bloque de constitucionalidad en salud, es preciso citar la Declaración Universal de los Derechos Humanos de 1948, en especial sus artículos 22 y 25; así mismo, el Pacto Internacional de Derechos Económicos, Sociales y Culturales de 1967, y de este, en especial, su artículo 12 y la observación 14 que lo desarrolla. Igualmente, es preciso citar la Declaración de Lisboa sobre los Derechos y Deberes de los Pacientes y el Código Iberoamericano de la Seguridad Social (2-5).

La concepción de la salud como un derecho fundamental autónomo fue tortuoso; sin embargo, en la actualidad se puede afirmar, sin lugar a duda, su fundamentalidad, no solo con base en las referencias jurisprudenciales al respecto, sino en la Ley Estatutaria de la Salud 1751 de 2015, por la cual se garantiza como derecho fundamental y se regulan y establecen sus mecanismos de protección (6,7).

Se resalta el artículo 1, objeto de esta ley, reconocer que las enfermedades huérfanas representan un problema de especial interés en salud, dado que, por su baja prevalencia en la población y su elevado costo de atención, requieren dentro del Sistema General de Seguridad Social en Salud (sGsss) un mecanismo de aseguramiento diferente al utilizado para las enfermedades generales, dentro de las que se incluyen las de alto costo, y unos procesos de atención altamente especializados y con gran componente de seguimiento administrativo.

También se resalta el artículo 2, "Denominación de las enfermedades huérfanas: artículo modificado por el 140 de la Ley 1438 de 2011”, que modifica el texto así: "Las enfermedades huérfanas son aquellas crónicamente debilitantes, graves, que amenazan la vida y con una prevalencia menor de 1 por cada 5.000 personas, comprenden, las enfermedades raras, las ultra-huérfanas y olvidadas" (8).

La Ley 1392 de 2010 había reconocido las enfermedades huérfanas de especial interés y adoptó normas tendientes a garantizar la protección social por parte del Estado colombiano a dicha población y sus cuidadores (9). Esta ley la modificó la ya mencionada Ley 1438 de 2011 (8).

Como complemento de la garantía del derecho a la salud, existe el sGsss, creado como parte del Sistema de Seguridad Social Integral, según lo establecido por la Ley 100 de 1993 y las leyes 1122 de 2007 y 1438 de 2011, que contienen múltiples normas reglamentarias al respecto $(8,10,11)$.

El sGsss se estructura como un servicio público para cubrir las contingencias de enfermedad general y maternidad y se divide en dos regímenes, a saber: el contributivo, dirigido hacia la población con capacidad de pago, y el subsidiado, dirigido hacia la población pobre y vulnerable, y ambos - en comienzo- deben garantizar el plan de beneficios en salud que, según los principios de la Ley 1751 de 2015, debe ser integral, aunque con base en el artículo 15 
de tal ley se han determinado exclusiones, principalmente, por la vía de resoluciones administrativas del Ministerio de Salud y Protección Social (7).

En referencia a la protección de la población que padece enfermedades huérfanas, en el caso de las personas con enfermedad de Huntington (ЕH), parece que el sGsss colombiano no logra materializar el goce efectivo del derecho a la salud; por el contrario, las múltiples barreras a las que se ven enfrentados hacen ilusoria la promesa constitucional del bienestar y de una vida digna como premisa de nuestro Estado social de derecho.

La EH consiste en un trastorno neurodegenerativo, trasmitido genéticamente y se caracteriza por una tríada de alteraciones: mentales, movimientos coreicos y deterioro cognitivo $(12,13)$. La persona nace sana y desarrolla la enfermedad generalmente en la mitad de la vida adulta, característicamente entre los 35 y los 55 años de vida, evoluciona crónicamente después de que inician los síntomas, entre 10 y 20 años hasta la muerte (14). En la actualidad, no se cuenta en el mundo con un tratamiento curativo; sin embargo, pueden recibir algunos tratamientos para los síntomas y terapias interdisciplinarias para aliviar su condición y la del cuidador que se ve comprometida, por lo que implica cuidar estas personas, cuya situación se podría resumir en tener, a la vez, enfermedad mental, trastornos del movimiento y demencia (15).

El objetivo del presente artículo es presentar la relación de las personas con EH con el sGsss en Colombia. Por tratarse de una enfermedad huérfana, el diagnóstico, el seguimiento y la prevención en generaciones futuras constituyen un desafío, tanto para las personas como para el personal de salud, como se reconoce en publicaciones de la Federación Española de Enfermedades Raras (16).

\section{Materiales y métodos}

$E_{r}^{1}$ estudio correspondió a una investigación cualitativa basada en los postulados de la teoría fundamentada, producto de la tesis doctoral en enfermería llamada La vida cotidiana de las personas con enfermedad de Huntington (17). Participaron 33 personas colombianas con Ен о a riesgo, con diferente sexo, condición socioeconómica, estado civil, religión y nivel educativo. Las técnicas de recolección de la información fueron: entrevistas semiestructuradas, observación, diarios de campo y revisión documental. Las entrevistas se hicieron después del estudio exploratorio, en el cual se examinaron las preguntas y se ajustaron los temas. Las entrevistas semiestructuradas fueron una virtual, vía Skype, y las demás cara a cara; tres de ellas en un consultorio y el resto en el lugar natural donde residían las personas. La duración fue entre una y dos horas. Hubo más de un encuentro con ocho participantes, lo cual permitió aclarar y profundizar la información; algunos familiares y cuidadores estuvieron presentes durante las entrevistas. Se complementaron los datos con entrevistas 
semiestructuradas y en conversaciones informales así: dos entrevistas a personas expertas, dos a cuidadores, tres a familiares de los participantes y tres a miembros de asociaciones de usuarios, dos de ellas, además de ser líderes, son personas a riesgo de enfermar (18). Ninguna persona se negó a participar. Todas las entrevistas fueron grabadas y transcritas. Se realizaron 350 horas de observación participante, también transcritas después de cada observación, para un total de 215 páginas de diario de campo (19). La revisión documental permitió estudiar la normatividad, la legislación y las estadísticas disponibles (20).

Fueron altamente representativos de los enfermos el Grupo de Investigación Neurociencias de Antioquia (GNA) y las fundaciones y asociaciones de usuarios de las regiones. Posteriormente al aval del Comité de Ética de la Investigación de la Facultad de Enfermería de la Universidad de Antioquia, acta CEI-FE 2017-5 del 27 de enero de 2017, se realizó la comunicación con los cuidadores para concertar las entrevistas. Se cumplieron los criterios éticos: la Declaración de Helsinki de la Asociación Médica Mundial (21), la Resolución 8430 del Ministerio de Salud colombiano y los siete criterios propuestos por Emanuel Ezequiel para que una investigación sea ética (21-23). Los testimonios se identificaron con la letra E, para entrevista, o Dc, para diario de campo, con las iniciales del participante y la fecha: mes, día, año. Se adicionaron sexo, edad y la localidad, para caracterizarlos mejor. Los nombres se modificaron por seudónimos para proteger la identidad.

Las trascripciones de las entrevistas y los diarios de campo se leyeron varias veces para la codificación abierta o microanálisis, del cual surgieron los códigos iniciales, organizados en Excel (17). Posteriormente, se hicieron agrupaciones de familias de códigos para llegar a las categorías. A través de la "recolección de datos, se hicieron comparaciones”, se buscaron diversos lugares, personas o acontecimientos y durante el análisis se buscó descubrir las variaciones (17).

La recolección y el análisis fueron concurrentes (17). En la codificación axial, se enlazaron las categorías y se buscó llegar a la comprensión de la relación de las personas con el sGsss (17). Se alcanzó saturación de datos con 33 participantes. Se hicieron devoluciones parciales antes de salir del campo y divulgaciones en cuatro eventos internacionales, uno nacional y dos regionales.

Los investigadores reconocen el efecto perturbador que la investigación produce en las familias y las comunidades. Con esa conciencia se tuvo presente la práctica de la reflexividad, que como expresa Cuesta-Benjumea "tiene un papel por el reconocimiento que hace nuestra presencia en la investigación, que si bien no se puede controlar, en el sentido positivista de la palabra, sí que se debe hacer lo más consciente posible” (24). La recolección de los datos en su lugar natural y el compromiso de los investigadores, "habla de su conexión con la situación”, tal como plantea la misma autora. La conciencia y la sensibilidad con las condiciones difíciles y las prácticas coercitivas y adversas de las instituciones del sistema de salud para obtener los servicios acercan este trabajo al modelo de reflexividad crítico-social (25). 
Se tuvieron presentes, especialmente, los siguientes criterios de rigor (26): credibilidad, por el tiempo en el campo y la transcripción textual y personal delas entrevistas; transferibilidad, por la presentación amplia del contexto que favorece los criterios de aplicabilidad de los resultados a otras enfermedades huérfanas y neurodegenerativas; auditabilidad interna con interlocución constante por asesores y auditabilidad externa con evaluadores durante la candidatura al doctorado y la evaluación final, y confirmabilidad en la interpretación de la información y la devolución a los participantes para confirmar los hallazgos (27).

\section{Resultados}

$\mathrm{L}$ as personas con EH sufren alteraciones de la marcha, con posibilidades de lesiones por caídas; del lenguaje, que se torna incomprensible y posteriormente se pierde; de la deglución de alimentos, o del comportamiento, con cambios en la personalidad, descuido, aislamiento social, agitación, agresividad, trastornos del sueño, depresión con intento de suicidio o suicidio consumado (28). Estas situaciones se acompañan de deterioro cognitivo con pérdida de memoria y orientación y dificultades para el desarrollo de las funciones básicas de la vida, como bañarse, vestirse, comer, entre otras, lo que les impide ser independientes. Así, entre la fase intermedia de la enfermedad y la final, se hace indispensable un cuidador, por el estado grave y progresivo de discapacidad.

El proceso inicia con cambios, como movimientos no controlados de alguna parte del cuerpo, que evolucionan a deterioro del lenguaje y la deglución, pérdida de peso, del empleo, en muchos casos de la familia, y discapacidad y dependencia completas hasta la muerte. Es una condición de deterioro que es vistosa y genera estigma social para los afectados, como plantea Goffman, por la "situación del individuo inhabilitado para estar en plena aceptación social" (29). Las personas reconocen que "Es una mezcla de... degenerativo de cuerpo y de mente” (E26 мн. Hombre mayor de 50 a. P. 3 Medellín).

Las implicaciones de la enfermedad dejan ver las circunstancias de su vida cotidiana, para asumir los desafíos para sobrevivir, pues, además del deterioro de la salud, enfrentan las situaciones de adversidad del sistema de salud y la pérdida del empleo. Generalmente, se trata de empleo informal que no genera cotización para obtener pensión de invalidez o, aun haciéndolo, por el hecho de enfermarse a edad temprana, no cuentan con las semanas necesarias de cotización para pensionarse por invalidez. Estas situaciones empeoran las condiciones de pobreza o los hace entrar en ella, e incrementa la precariedad de recursos necesarios para sobrellevar la situación. "Digamos que aquí la situación más compleja se ha presentado... si, por la parte financiera, porque yo no recibo ayuda del Estado ni nada... Nada, nada” (E05 LFD. Hombre mayor de 40 a. P. 287 Bogotá). 
La falta de recursos económicos se convierte en una de las situaciones que entorpece otras posibilidades, como la de ofrecerles seguridad y confort. Así relata este participante sus condiciones económicas y las necesidades insatisfechas que percibe:

Aún está pagando al banco las cuotas del apartamento en el que viven, la esposa que ahora está enferma, obtuvo la pensión por invalidez, él no tiene trabajo formal, vende en la casa electrodomésticos nuevos como ollas, planchas, licuadoras. El hijo adolescente está estudiando, con esa pensión no alcanza a pagar la deuda del apartamento, los servicios, el predial, la alimentación, el colegio del hijo y a eso se suman los costos de los pañales para su esposa, la alimentación complementaria como el Ensure, sin contar que no le alcanza para pagar otras deudas adquiridas, salieron del carro por falta de presupuesto, no es posible comprar nada extra, ni mejorarle la silla de ruedas a su esposa, pintar la casa o hacer algún arreglo. (Dc 042517 P. 19 Bogotá)

Estas circunstancias se suman a las complejas relaciones de amor y desamor que se tejen con familiares, vecinos y amigos, y al contexto particular en cada región, en algunas con carencia de saneamiento básico y escasez de alimentos. Aunque en cada localidad existen diferentes intervenciones del Estado, las asociaciones, en términos de apoyos para alimentación, vivienda y atención en salud, son insuficientes para resolver tanta adversidad.

En este contexto, se muestra cómo se construye la difícil relación de las personas con el sGsss, en el cual toman diferentes caminos para resolver la atención y los cuidados necesarios, para un afectado por la EH, como se muestra en la figura 1. 


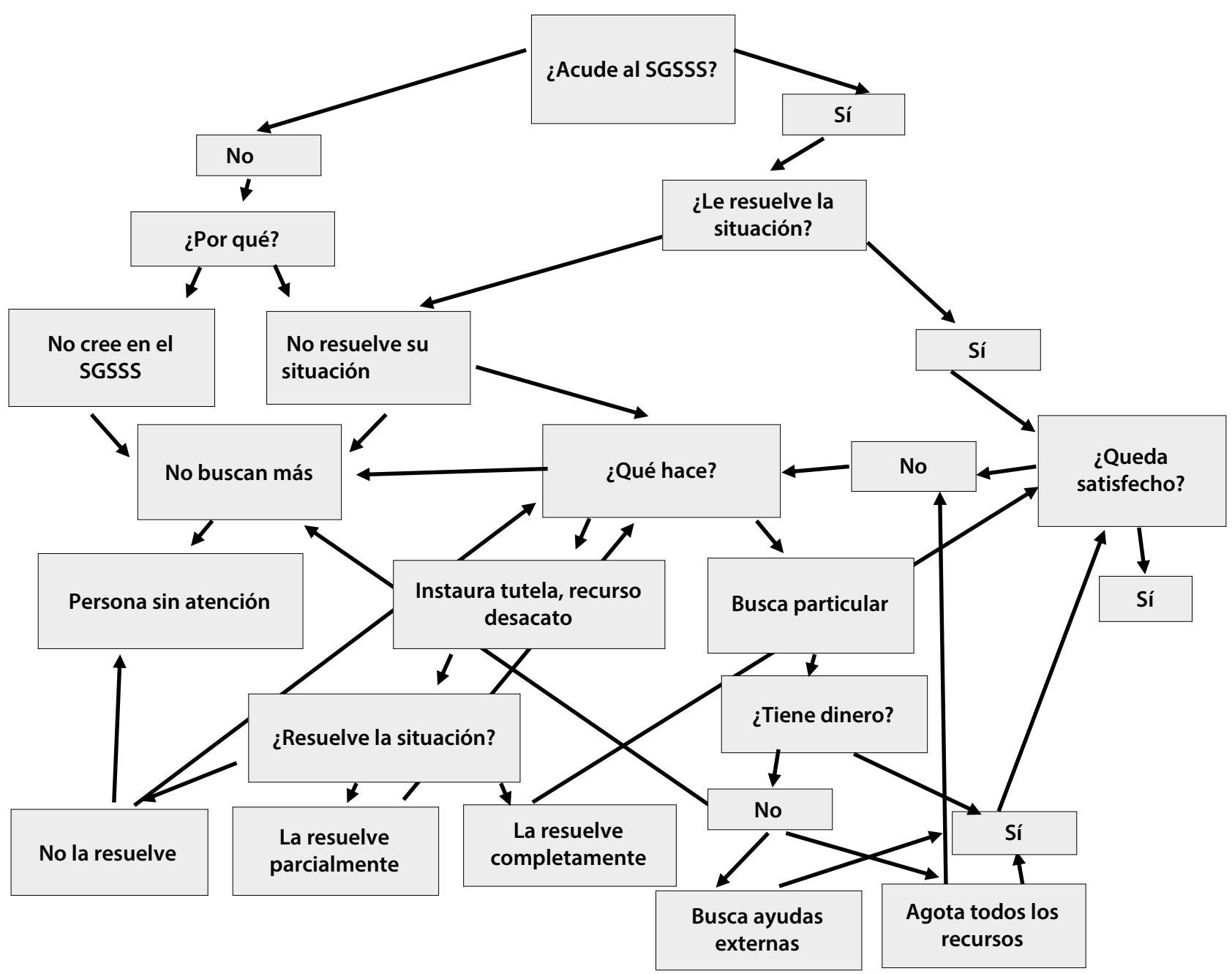

Figura 1. Posiciones de las personas con eH frente al sGsss en Colombia

Fuente: elaboración propia con base en trabajo de campo 2017-2018.

El esquema inicia con la pregunta: ¿acude usted al sGsss?, y de allí se desprenden las posiciones centrales: los que sí acuden y los que no lo hacen, cada grupo por diferentes circunstancias. Entre los que sí acuden se genera otra pregunta: ¿queda satisfecho? A lo que un grupo reducido responde que sí. Se trata de personas del régimen contributivo en las fases iniciales de la enfermedad, quienes reciben el diagnóstico por parte de un especialista de la empresa prestadora de servicios de salud (EPS) o por un grupo de investigación de un centro universitario que los remite a su EPs para la atención. Ellos se sienten satisfechos de lo que reciben. Así lo refiere la familia: “¿Qué EPs tiene Luis? xxxx, menos mal” (E09 LG. Hombre mayor de 50 a. P. 29 Medellín).

Aquellos quienes no quedan satisfechos optan por dos opciones: o buscar atención particular o emprender el proceso de la tutela integral, que consiste en un recurso jurídico, amparado en el artículo 86 de la Constitución Política de Colombia, "para reclamar ante los jueces, la protección inmediata de los derechos fundamentales, cuando estos resulten 
vulnerados o amenazados por la acción o la omisión de cualquier autoridad pública” (1). "Estando en la visita, llega a la casa el terapeuta respiratorio de Luis a atenderlo, en el cuarto donde estaba, había un compresor y una pipeta de oxígeno, una silla de ruedas eléctrica, estos recursos los lograron por la vía de la tutela” (DC FPG 042517 P. 15 Bogotá).

Frente a la búsqueda de atención particular, algunos cuentan con los recursos económicos para ciertos servicios: "Las terapias se las pagamos particulares, para poder mejorar la calidad de vida” (E01 JM. Mujer mayor de 50 a. P. 26 Bogotá).

Otros se debaten entre conseguir el dinero a toda costa o buscar ayuda. El pago de servicios o recursos de manera particular se abandona más temprano que tarde, por el alto costo y algunos deciden no buscar más y quedar sin atención, abandonados a su suerte, con todas las implicaciones de los síntomas de la enfermedad, sin recibir tratamiento. "Cuando teníamos medicamento para él, se le daba y estaba más tranquilo, pero ahora no tenemos medicamento; entonces con él es más difícil, quiere caminar y se cae, o se nos va de la casa, ya tocó pasarlo a otro cuarto porque no deja dormir” (Dc 110527 P. 25 Juan de Acosta).

Hay un grupo que agota todos los recursos, para seguirle la racionalidad al sistema, que se convierte para ellos en un antisistema, como lo refiere este participante:

Le toca a uno chuparse todo ese tiempo, esperando que llegue el primero (del mes) y si no le contestan, se tiene que ir uno para la, para la, ¿cómo es que es? Para la clínica, a sacar la cita. Entonces qué dicen: mira que no hay agenda... Este, la cita queda para el 27 del mismo mes, o sea, y entonces el proceso de ahí, a que lo atiendan a uno, el médico le formule el medicamento que uno tiene, que uno vaya a la EPs, que la EPS se lo apruebe, o sea, ya han pasado como cuatro meses en un solo proceso, entonces lo que aquí no entienden los familiares de los pacientes, ¿ya? Porque ellos no van a hacer las vueltas, las vueltas las hago yo. (La presidenta de la Fundación en Juan de Acosta). (E16 DE mujer mayor de 30 a. P. 19 Juan de Acosta)

La EH, como enfermedad rara, es de alto costo, no solo porque el tratamiento para los movimientos coreicos lo es, sino porque se requiere atención interdisciplinaria por parte de los servicios de neurología, psiquiatría, psicología, nutrición, enfermería, fisioterapia, trabajo social, entre otros, lo cual no está al alcance de las personas en sus lugares de residencia, lo que dificulta la atención. Dicha distancia implica costos adicionales para el desplazamiento, la alimentación y el acompañante, cuando la persona ha perdido la independencia, además del incremento de costos para el transporte individual, por las condiciones de deterioro. "En la condición en que ella está, ya viajar es imposible, o sea es muy difícil, salvo que tuviera muy buenos recursos y me la lleve a un hotel donde tenga todo, digamos que hice hasta lo último, hasta lo último, ya cuando podía llevarla ehh, pero ya me empezó a generar inconvenientes” (E07 LFD hombre mayor de 40 a. P. 25 Bogotá). 
De los que acuden al sgsss por vía de la tutela, algunos también la abandonan por los múltiples procesos: filas, visitas médicas y repetición de trámites para allegar la documentación médica requerida y que se falle a su favor. "La gente también es conformista ¿no?, o sea, tal día empieza como a hacer las vueltas, les molesta, finalmente hacen algunas y después ya no hacen más, pero dicen: ¿pero si no hay cura? Y tienen razón, no tiene cura” (E01 JM mujer mayor de 50 a. P. 14 Bogotá).

Otros, de manera resiliente, desarrollan todo el proceso con atajos, adversidades y reprocesos para lograr la atención integral por esta vía. Ellos están ubicados en la capital o en ciudades principales, conocen la ruta porque alguien los guía, como un abogado, un familiar o los líderes de las asociaciones de usuarios. "Le pido que haga la tutela, incluya las leyes, le pido por favor que busque que la vea el neurólogo, para que le puedan dar todo, porque el neurólogo es el que debe formular” (E01 Jm. Mujer mayor de 50 a. P. 40 Bogotá).

Algunos, aun con tutela, no logran los beneficios. No solo es necesario que esta falle a su favor, sino que el asegurador cuente con el prestador de los servicios que el paciente requiere. "La tutela salió con fallo a favor de mi esposo, nos han entregado dosis de diciembre y enero, supuestamente las entregas eran entre enero y marzo, hasta la fecha no hay entrega (noviembre), según ellos no hay en la farmacia” (E01 Jм mujer mayor de 50 a. P. 24 Bogotá).

Entre el grupo que persiste se observa fortaleza, según ellos, movida por el amor a su familiar, que los motiva a vencer cualquier obstáculo. Conocen la norma, los tiempos, el lugar de radicación de la documentación y se muestran empoderados de tal manera que conocen el paso a seguir en caso de negación de los servicios, y cuando la tutela ha fallado a su favor, interponen recursos de petición, desacato o un movimiento en los medios de comunicación o en las redes sociales hasta obtener los servicios. "Haga la tutela, repórtela, y copie a la Supersalud, además de la Personería, la Procuraduría y la misma eps a la que corresponda” (E01 JM mujer mayor de 50 a. P. 10 Bogotá).

Otros renuncian al proceso y su familiar queda sin atención o con la mínima que se logre alcanzar. Entre tanto, aprovechan las intervenciones de los grupos de investigación de las universidades o las asociaciones, que les ofrecen atenciones puntuales. "Cuando vienen las brigadas, ¿ellos qué les dan? Le dan a uno fórmula, si tienen la pastilla enseguida la dan... La vez pasada la llevé a psicología, nutrición, terapia, la llevé a nutricionista, le hicieron exámenes, con practicantes de la universidad" (E11 oE mujer mayor de 50 a. P. 42 Juan de Acosta).

Entre los que no acuden al sgsss se encuentran los que no creen en él o piensan que este no resuelve nada, porque conocen la complejidad de la enfermedad y su condición de incurable, lo que para algunos es una lucha sin posibilidad de salvación:

Qué vamos a ir a esa reunión, si el mismo cuento... Ya vienen a molestar na más... Ya la gente como que se cansa de eso... Se han vuelto apáticas, porque si las personas de Huntington vieran que hubieran hecho de pronto una oficina y los enfermos fueran allá, o los familiares a buscar los medicamentos, se les dieran, no, no, no se hace nada... 
Ya la gente no quiere. Ellos siguen viviendo ahí su vida hasta que Dios quiera, porque medicamentos hasta ahora mismo no, no toman los enfermos de Huntington. (E17 IM hombre mayor de 50 a. P. 20 Juan de Acosta)

Aquí se observa lo que el psicólogo Seligman tipificó como desesperanza aprendida, no emprender acciones para lograr servicios de salud, porque nada va a funcionar. Dicha creencia está fundamentada en las fallidas experiencias previas, propias o de otras personas. Así lo explica Seligman, "la desesperanza aprendida es un proceso cognoscitivo en el cual la formación de expectativas se ve afectada por experiencias previas en las que se reciben castigos o tratos arbitrarios, independientemente de la conducta” (30).

Este grupo generalmente se encuentra en regiones apartadas, como Bellavista (Chocó) —el lugar donde se reconstruyó Bojayá posterior a la masacre del 2012 — o en Juan de Acosta (Atlántico), municipio paradigmático por ser la segunda región del mundo con el mayor número de afectados de EH, después del lago de Maracaibo, en Venezuela (31).

En estas poblaciones, de baja escolaridad, escasos recursos económicos, escasez de servicios públicos e, incluso, necesidades básicas insatisfechas, la enfermedad implica un proceso que empeora las condiciones de precariedad. Así lo relata una participante:

Los dejan en un cuarto oscuro, en colchones en el piso, porque ellos se caen de la cama, les tiran trapos, eso huele muy mal, con esos calores y sin abanicos, allá les ponen la comida, ellos a veces están muy agresivos, nosotros tratamos de conseguir colchones nuevos, pañales, abanicos, comida, pero ahora la presidenta (de la asociación de usuarios) que está, no gusta de mí. (Dc 062517 P. 3 Juan de Acosta)

Las condiciones de baja escolaridad, distancia de los servicios y escasos recursos económicos muestran el efecto de la injusticia social, lo que se denomina en salud colectiva determinación social, que releva:

[...] las relaciones de poder y exalta las dinámicas de acumulación de capital como esenciales para entender los procesos salud-enfermedad, que conllevan los patrones de trabajo y de consumo, las falencias de los soportes sociales y las formas de cultura que conducen a formas malsanas de vivir y llevan a los individuos a padecer la enfermedad de forma diferencial, según clase social, género o etnia. $(32,33)$

En cada región es diferente la manera como opera el sistema de salud, aunque sea un mismo asegurador, esto es, no funcionan igual los prestadores en los municipios y en las ciudades principales. Hay diversidad en la atención según la red de prestadores con los cuales cuente. Las ciudades principales y la capital Bogotá parecen contar con mejores prestadores que los lugares más apartados, por lo que la atención es mejor en las primeras y muy mala en los segundos. 
La responsabilidad de la salud de las personas está diluida entre el Estado, la aseguradora y los prestadores, es decir, está en tierra de nadie, porque cada uno elude la responsabilidad que debería cumplir.

Algunas personas se ven empoderadas sobre sus derechos y la legislación, pero esto es insuficiente para obtener los servicios. Al final de cuentas, todas las personas que desarrollan la EH mueren en un proceso crónico de deterioro de 10 a 20 años; sin embargo, sus condiciones de vida son bastante distintas según el contexto.

El esquema que ilustra las situaciones expuestas se acerca a lo que Clarke et al. denominan mapa de análisis situacional y expresan: "las diferentes posiciones que las personas toman frente a una misma situación” (34); en este caso, la de los participantes frente al sistema de salud colombiano.

\section{El Sistema General de Pensiones}

El Sistema de Seguridad Social Integral, creado por la Ley 100 de 1993, estructuró tres sistemas o subsistemas: el Sistema General de Pensiones, el sgsss y el Sistema General de Riesgos Laborales. También la misma Ley 100, en su artículo 279, excluyó de su aplicación a algunas poblaciones hoy denominadas regímenes exceptuados, entre ellas: la Policía, los militares y el magisterio público que, en general, poseen sus propios sistemas de pensión, de acceso a la salud o de ser objeto de protección de los riesgos laborales.

En el caso de las personas con ЕH, es escaso que alcancen una pensión de vejez, por ser un sistema básicamente de tipo contributivo, es decir, de aportes y cotización, y las personas no logran responder a estos pagos por la alta informalidad de su trabajo, entre otros. Para los pocos que logran la pensión de invalidez, esta puede mitigar la situación económica de la familia. Un participante que obtuvo su pensión por el accidente que sufrió mientras prestaba el servicio militar expresó que de su pensión depende económicamente su familia: "Me dice que perdió su pierna derecha en el Ejército, hace mucho tiempo, que le dieron la prótesis, pero nunca se logró adaptar a ella y que maneja muy bien las muletas, por ser lesionado en el Ejército, lo pensionaron y eso es una gran ayuda” (DC 110517 P. 13 Juan de Acosta).

Aquellos quienes tuvieron un trabajo formal, al enfermarse, con pocos años de vida laboral, no cumplieron el número de semanas cotizadas para acceder a pensión. El siguiente es el caso de una mujer, médica, que inicia los síntomas a los 36 años y debe retirarse del trabajo: "Una cosa muy importante en mi vida, se me olvida... La pensión me la negaron, entonces como tengo la pensión negada, entonces me dan solamente 500000 pesos... En un СDT... (Le dieron un bono pensional que es el monto del dinero que tiene en cDT, del cual le daban \$500000 mensuales en el 2017)” (E03 LEM mujer mayor de 50 a. P. 9 Medellín).

Este dinero correspondía, en su momento, a menos de un salario mínimo legal en Colombia, que para ese año era de 737717 pesos. Aunque vivía en la casa con su madre, 
este monto no lograba satisfacer sus necesidades; sin embargo, aportaba a los gastos. Así lo expresa:

De ahí saco la plata pa comprar el almuerzooo, el desayuno es cafecito o Milo con parva, el almuerzo sí es compraito... La tranquilidad es de uno poder saber, que con un plato de 14000 (risas) pesos, comen dos, muy grande, entonces el de las dos, compramos uno para las dos (ella y su mamá). (E03 LEM mujer mayor de 50 a. P. 29 Medellín)

Otra de las personas que no logró pensión de invalidez recibió la devolución del bono pensional. Esto relata la participante, sobre la situación de su hermana:

Bueno entonces yo la pensé, yo dije: pero ella no va a gozar esa pensión, porque allá en Barranquilla es una mamadera de gallo del seguro, que una cosa que otra, entonces yo dije: no, yo hablé con mi hija, nos vamos a hacer esto, vamos a ir al seguro pa ve si la, si la indemnizan, eso fue un proceso grandísimo... Mi hija fue y cobró lo único que le dieron (de bono pensional) a ella, de 28 años (de trabajo) fueron 13 millones (En el año 2012). (E07 Ev mujer mayor de 50 a. P. 53 Chocó)

En otros casos, la pensión está en trámite sin tener claridad respecto a si se podrá lograr o no:

Ah, no me han pensionado, ahí estoy. ¿Estás cotizando? Ya no, ya aquí la doctora le dijo... Le dio unos documentos donde... Nos colaboraron mucho, y en el momento estamos en el proceso porque esta como difícil que porque a él... Me faltan semanas. Le faltan semanas... 50 semanas de cotización, le faltó semanas, quedó en la mitad. Como le digo yo doctora, a él que lo que hay que hablar, es porque a él no lo van a pensionar por vejez, sino por invalidez. (E09 LG hombre mayor de 50 a. P. 23 Medellín)

Sobre los procesos legales, tan indispensables para estas personas, ni los afectados ni sus cuidadores tienen claridad, por lo que requieren, en la mayoría de los casos, una intervención profesional que está fuera de su alcance. "Es que yo volví a C. ¿Cuándo fue, mija? Antier y me dijeron lo mismo: que el abogado, que hay que esperar que me llamen, ¿de dónde fue? Él necesita como otro papel, doctora. No me hizo la evaluación el médico, y otra carta y ya vuelvo a S. a C.” (E09 LG hombre mayor de 50 a. P. 42 Medellín).

El puntaje de calificación de invalidez le otorga una esperanza a esta familia: "Mire que a él le salió muy buen puntaje, le salió 76.65 (de invalidez), imagínese que la doctora quedó asustada. Dijo: ‘No pues ¿qué más van a buscar?’”. (E09 LG hombre mayor de 50 a. P. 43 Medellín). Sin embargo, esto hace parte de los requisitos, pero no son suficientes para obtener la pensión por invalidez. En otra circunstancia, la hija de una persona afectada de eH busca lograr la pensión de un hermano que ha fallecido, para favorecer a su madre. "Mi hermanito (que falleció) está en proceso de pensión y estoy por medio de un abogado, para reclamarla para ella (la madre) y mi papá” (E01 JM mujer mayor de 50 a. P. 40 Bogotá). 
La mayoría no tiene ninguna esperanza de pensión. Se caracterizan por empleo informal o por su ausencia, bien por la oferta escasa para las funciones que pueden realizar, según la vocación comercial, en ganadería o agricultura, o porque sus capacidades se han deteriorado por la enfermedad. "Este hombre blanco, en quien se ven en sus piernas las evidencias de las picaduras de mosquitos, era el proveedor de la casa. Hoy día está enfermo, ya no hay un proveedor, vive con su esposa, también adulta mayor, en esta finca” (Dc 110517 P. 23 Juan de Acosta).

Otra queja de quienes pueden laborar es la experiencia de verse desplazados por mano de obra de venezolanos migrantes, que realizan la misma actividad por menos dinero. "El trabajo ha estado muy malo, entre otras cosas, porque han llegado venezolanos que están haciendo el trabajo por la mitad del precio” (DC 110517 P. 5 Juan de Acosta).

El tipo de empleo por el cual compiten sigue siendo en la informalidad: empleo doméstico por días, en oficios de construcción o del campo o como venteros ambulantes. "La participante me relata: mi otra hija también vive en Barranquilla, trabaja en una casa, pero el trabajo es muy pesado y está muy aburrida, espera venirse en diciembre para la casa, ella es la que me ayuda para la comida y la de las niñas” (Dc 110517 P. 19 Juan de Acosta). "Yo también he trabajao en los buses (vendiendo dulces), yo también he vendido en los buses” (E10 zc mujer mayor de 30 a. P. 31 Medellín).

Como se refirió, las condiciones de pobreza son una constante en las personas con EH, que bien podría llevar el calificativo de enfermedad catastrófica o ruinosa, de acuerdo con la Ley 972 de 2005 (35), que la define como “aquellas que representan una alta complejidad técnica en su manejo, alto costo, baja ocurrencia y bajo costo efectividad en su tratamiento” (36).

La complejidad técnica de la $\mathrm{eH}$ tiene que ver con la dificultad para acercar a las personas comprometidas con el equipo interdisciplinario que requieren para la atención de las múltiples y complejas alteraciones derivadas de la enfermedad, a lo que se suma el alto costo de los medicamentos, la baja ocurrencia (por tratarse de una enfermedad huérfana) y el bajo índice costo efectividad, ya que no existe en la actualidad un tratamiento curativo. Así ven los otros a los afectados por la EH: "No es fácil, y es un sufrimiento grande en el cual viven todas estas familias (en El Difícil, Atlántico), en las mismas condiciones de pobreza, de abandono de estigmatización, de exclusión, o sea, no merecen vivir como están... Viviendo, pues nadie es libre de tener una enfermedad tan terrible como esta” (E23 мc mujer mayor de 50 a. P. 7 Santa Marta).

\section{Discusión}

as personas con ен en Colombia requieren atención especializada e interdisciplinaria para
hacer frente a la tríada de alteraciones que presentan: el trastorno mental, los movimientos 
coreicos y las alteraciones cognitivas. Entre los efectos de la enfermedad están los cambios del estado de ánimo y del comportamiento, la depresión y el intento suicida, las caídas y la broncoaspiración, por las dificultades para la deglución que los puede llevar a la muerte.

El proceso de la enfermedad tiene cambios, pérdidas y deterioro hasta la discapacidad; por tanto, en todos los casos es necesario un cuidador. Al perder el empleo, los pocos que lo tenían de manera formal no logran la pensión de invalidez y los que laboraban en la informalidad entran en mayor pobreza y escasez, lo que empeora sus condiciones de vida. Las personas que buscan atención en el sGsss encuentran barreras de todo tipo, derivadas de la falta de accesibilidad y de información y, por supuesto, de las dificultades económicas. Algunos buscan superar las dificultades de acceso a través de la tutela, y quienes persisten están empoderados de sus derechos y son residentes en ciudades principales o intermedias del país.

Es de resaltar la importancia de la acción de tutela en todo el proceso de búsqueda de la garantía y del goce efectivo del derecho fundamental a la salud de las personas con enfermedades huérfanas. La acción de tutela fue consagrada en el artículo 86 de la Constitución Política de Colombia de 1991 y reglamentada básicamente por el Decreto Ley 2591 de 1991, y como lo demuestran múltiples informes defensoriales, las tutelas han sido casi la única vía eficaz de protección de la salud integral de dichas personas y de acercamiento a la justiciabilidad de su derecho y del acceso a las prestaciones asistenciales y económicas derivadas (1,37).

Frente a las mismas barreras, otro grupo de regiones distantes, como Chocó y Juan de Acosta, emprenden pocas o ninguna medida, en una suerte de desesperanza aprendida, como bien tipificó Seligman (30). Ellos presentan la enfermedad en su estado genuino, porque no reciben ninguna atención y sus condiciones son precarias hasta la desnutrición y el abandono.

La EH es una enfermedad crónica, compleja, catastrófica y ruinosa, y los afectados y sus familias no logran satisfacer sus necesidades, las cuales se incrementan en insumos para el cuidado como pañales, complementos nutricionales, silla de ruedas, transporte especial y el mismo cuidador.

En esta investigación se puede observar el efecto de lo que en el campo de la salud colectiva se denomina determinación social, es decir, la manera diferencial como se vive según clase social, género o etnia (33). Tener eH genera estigma, por los cambios corporales imposibles de ocultar, lo cual vulnera aún más a los afectados y a sus familias (29). Frente a una enfermedad hereditaria como esta, es necesario generar estrategias y acciones afirmativas, educativas, de prevención para las próximas generaciones, difundir las rutas de atención y de asistencia a estas personas y a sus cuidadores y empoderarlos sobre sus derechos y la legislación para su atención, que nos acerque al cumplimiento de los objetivos de sostenibilidad $(38,39)$.

En general, la relación entre los pacientes con EH, sus cuidadores y el sGsss colombiano, en voces de los pacientes y sus familias, no es una relación ni armónica, ni efectiva. Casi podríamos afirmar que el sGsss y sus múltiples articulaciones de actores y normas se han erigido más como una barrera que como un camino de bienestar y de esperanza para ellos. 
Barreras que, muchas veces, no logran romper con la interposición de acciones de tutela. La promesa de un derecho fundamental e integral para las personas con EH y sus cuidadores y familias parece quedarse solo en el terreno de lo formal y no logra la materialización real del goce efectivo de la dimensión de servicio público esencial de la salud en un Estado social que debería garantizar tal derecho.

\section{Agradecimientos}

\footnotetext{
A los participantes de la investigación, sus familias y cuidadores, por hacer posible este 1 trabajo; a la PhD Sonia Moreno, neuropsicóloga del Grupo de Neurociencias de Antioquia y a su equipo, a la Asociación Colombianos por la Enfermedad de Huntington, a la Asociación de Comunidades Vulnerables de Colombia, a la Fundación de Personas con Enfermedad de Huntington de Juan de Acostas y a la Universidad de Antioquia, por conceder la comisión para el estudio a una de las investigadoras.
}

\section{Contribución de los autores}

Todos los autores contribuyeron en las diferentes fases de la investigación y revisaron y
aprobaron el texto final del artículo.

\section{Conflicto de intereses}

Ninguno declarado.

\section{Referencias}

1. Colombia. Constitución política. Bogotá; 1991. p. 108.

2. Declaración Universal de Derechos Humanos. París: Asamblea General de Naciones Unidas; 1948.

3. Pacto Internacional de Derechos Económicos, Sociales y Culturales. Nueva York: Asamblea General de Naciones Unidas; 1966.

4. Declaración de Lisboa. Portugal: Asociación Médica Mundial sobre los Derechos del Paciente; 1988. 
5. Código Iberoamericano de la Seguridad Social. Madrid: Organización Iberoamericana de Seguridad Social; 1995.

6. Gañán Ruiz JL. De la naturaleza jurídica del derecho a la salud en Colombia. Estud Derecho [internet]. 2011;68(151):189-212. Disponible en: http://tesis.udea.edu.co/dspace/ bitstream/10495/2266/1/De la naturaleza jurídica del derecho a la salud en Colombia.pdf

7. Ley Estatutaria 1751/2015 del 16 de febrero, por medio de la cual se regula el derecho fundamental a la salud y se dictan otras disposiciones [internet]. Congreso de la República. Disponible en: https://www.minsalud.gov.co/Normatividad_Nuevo/Ley\%20 1751\%20de\%202015.pdf

8. Ley $1438 / 2011$ de 19 de enero, por medio de la cual se reforma el Sistema General de Seguridad Social en Salud y se dictan otras disposiciones [internet]. Diario Oficial 51.544, de 31-21-2020. Disponible en: http://www.secretariasenado.gov.co/senado/basedoc/ ley_1438_2011.html

9. Ley 1392/2010 del 2 de julio, por medio de la cual se reconocen las enfermedades huérfanas como de especial interés y se adoptan normas tendientes a garantizar la protección social por parte del Estado colombiano a la población que padece de enfermedades huérfanas y sus cuidadores [internet]. Congreso de Colombia. Disponible en: https:// www.minsalud.gov.co/sites/rid/Lists/BibliotecaDigital/RIDE/DE/DIJ/ley-1392-de-2010.pdf

10. Ley 100/1993 del 23 de diciembre, por la cual se crea el Sistema de seguridad social integral y se dictan otras disposiciones [internet]. Diario Oficial 41.148 del 23-12-1993. Disponible en: http://www.comisionseptimasenado.gov.co/salud/SALUD EN LEY 100 DE 1993.pdf

11. Ley 1122/2007 del 9 de enero, por la cual se hacen algunas modificaciones en el Sistema General de Seguridad Social en Salud y se dictan otras disposicoines [internet]. Diario Oficial 51.544 de 31-12-2020. Disponible en: http://www.secretariasenado.gov.co/senado/ basedoc/ley_1122_2007.html

12. Cattaneo E, Rigamonti D, Zuccato C. El enigma de la enfermedad de Huntington. Sci Am Mex. 2002;1(8):32-7.

13. Rodríguez Pupo JM, Díaz Rojas YV, Rojas Rodríguez Y, Rodríguez Batista Y, Núñez Arias E. Actualización en enfermedad de Huntington. Gen Univ Vladimir Ilich Lenin. 2013;17(1):546-57.

14. Nance M, Paulsen J, Rosenblatt A, Wheelock V. Guía para médico sobre el manejo de la enfermedad de Huntington [internet]. 3. ${ }^{a}$ ed. Sociedad de la Enfermedad de Huntington de América; 2011. Disponible en: http://hdsa.org/wp-content/uploads/2015/03/14_Guiapara-Medico-sobre-el-Manejo-de-la-enfermedad-de-Huntington..pdf

15. Yu M, Tan K, Koloms K, Bega D. Assessment of caregiver burden in Huntington's disease. J Huntingtons Dis. 2019;8(1):111-4.

16. Ruiz García B, Delgado González C, Cruz González J, Vasermanas D, Gutiérrez Alonso MI, Mayoral Rivero E, et al. Guía de apoyo psicológico para enfermedades raras [internet]. Madrid: Federación Española de Enfermedades Raras; 2009. p. 80. Disponible en: http:// www.ahuce.org/Portals/0/Publicaciones/Psicologia/Gu\%C3\%ADa\%20de\%20apoyo\%20 psicol\%C3\%B3gico\%20para\%20EERR.FEDER.pdf 
17. Strauss A, Corbin J. Bases de la investigación cualitativa: técnicas y procedimientos para desarrollar la teoría fundamentada. Medellín: Editorial Universidad de Antioquia; 2002.

18. Patton MQ. Qualitative interviewing. En: Qualitative evaluation and research methods. Newbury Park: Sage; 1990. p. 339-429.

19. Guber R. La etnografía: método, campo y reflexividad [internet]. Buenos Aires: Siglo Veintiuno; 2011. Disponible en: https://abacoenred.com/wp-content/uploads/2016/01/ etnografi-a-Me-todo-campo-reflexividad.pdf

20. Vélez Restrepo OL, Galeano Marín ME. Investigación cualitativa: estado del arte. Medellín: Centro de Investigaciones Sociales y Humanas, Universidad de Antioquia; 2002.

21. Asociación Médica Mundial. Declaración de Helsinki: principios éticos para las investigaciones médicas en seres humanos. An Sist Sanit Navar. 2001;24(2):209-12.

22. Resolución 8430/1993 del 4 de octubre, por la cual se establecen las normas científicas y administrativas para la investigación en salud [internet]. Ministerio de Salud. Disponible en: https://www.redjurista.com/Documents/resolucion_8430_de_1993.aspx\#/

23. Emanuel E. ¿Qué hace que la investigación clínica sea ética? Siete requisitos éticos. En: Pellegrini Filho A, Macklin R, editores. Investigación en sujetos humanos: experiencia internacional. Santiago de Chile: Programa Regional de Bioética ops/oms; 1999. p. 33-46.

24. Cuesta-Benjumea $\mathrm{C}$ de la. La reflexividad: un asunto crítico en la investigación cualitativa. Enferm Clin. 2011;21(3):163-7. https://doi.org/10.1016/j.enfcli.2011.02.005

25. Finlay L, Gough BE. Reflexivity: a practical guide for Researchers in health and social sciences. London: Wiley-Blackwel; 2003.

26. Guba EG. Criterios de credibilidad en la investigación naturalista. 3. a ed. Madrid: Akal, 1989.

27. Arias Valencia MM, Giraldo Mora CV. El rigor científico en la investigación cualitativa. Investig Educ Enferm. 2011;29(3):500-14.

28. Kachian ZR, Cohen-Zimerman S, Bega D, Gordon B, Grafman J. Suicidal ideation and behavior in Huntington's disease: systematic review and recommendations. J Affect Disord. 2019;250(December 2018):319-29. https://doi.org/10.1016/j.jad.2019.03.043

29. Goffman E. Estigma la identidad deteriorada. Buenos Aires: Amorrortu; 2006.

30. Flórez Muñoz VR, González Salamanca R del P, Isaza de Gil G. Desesperanza aprendida: categoría emergente de los docentes frente a la inclusión educativa. Plumilla Educ. 2015;16(2):202-20.

31. Cubo E. La enfermedad de Huntington: un recorrido a través de la historia. Neurosci Hist [internet]. 2016;4(4):160-3. Disponible en: http://nah.sen.es/vmfiles/abstract/ NAHV4N42016160_163ES.pdf

32. Morales-Borrero C, Borde E. ¿Determinación social o determinantes sociales?: diferencias conceptuales e implicaciones praxiológicas. Rev Salud Pública [internet]. 2013;15(6):797808. Disponible en: http://www.scielosp.org/pdf/rsap/v15n6/v15n6a03.pdf

33. Casallas Murillo AL. La medicina social-salud colectiva latinoamericanas: una visión integradora frente a la salud pública tradicional. Rev Ciencias la Salud. 2017;15(3):397408. http://dx.doi.org/10.12804/revistas.urosario.edu.co/revsalud/a.6123 
34. Clarke A, Friese C, Washburn R. Situational analysis in practice: mapping research with grounded theory. Left Coast Press; 2016.

35. Ley 972/2005 del 15 de julio, por la cual se adoptan normas para mejorar la atención por parte del Estado Colombiano de la población que padece de enfermedades ruinosas o catastróficas, especialmente el viн/sida [internet]. Diario Oficial 45.970. Disponible en: https://www.minsalud.gov.co/sites/rid/Lists/BibliotecaDigital/RIDE/DE/DIJ/Ley_0972_ de_2005.pdf

36. Quintero Lyons JDC, Correa Solano L. Enfermedades catastróficas en el sistema general de seguridad social en salud en Colombia. Rev Jurídica Mario Alario D’ Filippo. 2009;1(2):9-27.

37. Decreto Ley 2591/1991 del 19 de noviembre, por el cual se reglamenta la acción de tutela consagradas en el artículo 86 de la Constitución Política [internet]. Diario Oficial 51.544 de 31-12-2020. Disponible en: http://www.secretariasenado.gov.co/senado/basedoc/decreto_2591_1991.html

38. Corte Constitucional de Colombia. Sentencia T-330/93, trato diferencial positivo [internet]. Disponible en: http://www.corteconstitucional.gov.co/relatoria/1993/T-330-93.htm

39. Naciones Unidas. Informe de los Objetivos de Desarrollo Sostenible 2017. Nueva York; 2017. https://doi.org/10.18356/70388b69-es 